\title{
ENRIQUE DUSSEL Y EL PENSAMIENTO CRÍTICO DE LA LIBERACIÓN
}

\author{
Abdiel Rodríguez Reyes \\ Universidad de Panamá \\ abdielarleyrodriguez@hotmail.com
}

RESUMEN: Este artículo analiza la producción teórica del pensamiento crítico de la liberación de Enrique Dussel. Plantea algunas dificultades de aproximarse a su obra. También algunos temas y debates que surgen a partir de su crítica a la modernidad.

Palabras clave: Filosofía, política, ética, pensamiento, crítica.

\section{ENRIQUE DUSSEL AND THE CRITICAL THINKING OF LIBERATION}

ABSTRACT: This article examines the theoretical production of critical thinking of liberation of Enrique Dussel. Poses some difficulties of approaching his work. Also some topics and debates that arise from criticism of modernity.

Keywords: Philosophy, political, ethics, thinking, criticism.

Recibido: 7 de Mayo de 2016 Aceptado: 7 de Julio de 2016

Los pensadores críticos latinoamericanos tienen que asumir dos funciones, por un lado, la interpretación de la realidad, y por otro, la militancia política con el ideal de las transformaciones sociales, un tanto marcados por la undécima tesis de Marx sobre Feuerbach que dice: "los filósofos no han hecho más que interpretar el mundo, pero de lo que se trata es de transformarlo." En los pensadores críticos latinoamericanos solo una brizna separa lo teórico de lo práctico, hay una simbiosis, dando paso a una praxis, en el caso que nos ocupa en este artículo, una praxis de la liberación desde el pensamiento crítico de Enrique Dussel. 
En el siglo XXI, pese a que muchos vientos están en contra del pensamiento crítico, habrá que tomar la dirección del ángel de la Historia que vio Walter Benjamin en su cuadro de Paúl Klee (Ángelus Novus). En este ir a contracorriente, la Filosofía de la Liberación ha aportado de manera significativa, movimiento del que forma parte el autor del cual nos ocuparemos en este artículo, que desde una perspectiva interdisciplinar aborda la cuestión del pensamiento crítico de la liberación.

\section{Enrique Dussel: autor y obra}

Enrique Dussel es mundialmente conocido por sus trabajos en el campo de la Ética, Filosofía, Política (y una Lógica en proceso) de la liberación. Para aproximarse a su obra, aunque solo sea de forma introductoria, debemos conocer mínimamente algunos datos del autor, no solo para hacer justicia a su vivencia como pensador, sino para conocer la evolución de su pensamiento en contexto. La biografía forma parte de la obra. Muchas veces el pensamiento está arraigado a lo vivencial.

Habrá que tomarse esto muy en serio, ya que la obra de Dussel no es un pensamiento que emerge de lo abstracto, sino de la materialidad, de los sujetos de carne y hueso que sufren; pensamiento que se eleva a la especulación dialéctica, hasta retornar en propuestas concretas. Cabe decir que de este filósofo hablaremos por muchos años, ya sea para acercarnos críticamente o alejarnos de su programa filosófico. Lo importante, al menos en este artículo, es entender la crítica como bifurcación entre todo el trabajo realizado y las tareas venideras, en particular para las nuevas generaciones de pensadores que se inician en la afanosa empresa del pensamiento crítico de la liberación.

En la obra de Dussel se mezclan continuamente diversos ámbitos del saber, podemos decir que son en la siguiente cadena de interrelaciones: Historia $\leftrightarrow$ Teología $\leftrightarrow$ Ética $\leftrightarrow$ Filosofía $\leftrightarrow$ Economía $\leftrightarrow$ Política $\leftrightarrow$ Lógica. Pero siempre intercomunicándose uno con otro, es un trabajo interdisciplinario en el sentido estricto de la palabra. Es muy difícil periodizar su obra en ejes temáticos estáticos, ya que es recurrente volver sobre ellos, toda su obra está en permanente movimiento.

\section{Perfil académico}

El perfil académico de Enrique Dussel nos ayuda a tener elementos cuantitativos y cualitativos para valorar su obra. En este epígrafe vamos a mencionar algunos datos. Dussel tiene dos licenciaturas (Filosofía y Estudios de la Religión) y dos doctorados (Filosofía e Historia). Es Doctor Honoris Causa de varias universidades latinoamericanas y europeas. En cuanto a su trayectoria docente, es profesor desde la década del setenta que se exilió en México -después de sufrir un atentado en Argentina-, en la Universidad Autónoma Metropolitana (UAM) y en la Universidad Nacional Autónoma de México (UNAM). Es profesor emérito en la UAM e Investigador Nacional Emérito del Sistema Nacional de Investigadores 
(CONACYT-México). Es decir, posee las máximas distinciones y ejecutorias con las que se le puede reconocer la trayectoria académica a un profesor universitario. También fue rector interino de la Universidad Autónoma de la Ciudad de México (Cf. Dussel, 2015: 355-364). Ha sido profesor invitado en más de una veintena de universidades, entre las que cabe destacar: Université de Fribourg, Goethe Universität, Duke University, Harvard University, entre otras. Ha dictado conferencias en todos los continentes, en decenas de universidades.

\section{Obra}

Para ver los detalles de la obra, Moreno (1998) realizó un excelente trabajo en el número 180 de la revista: Anthropos. Huellas del Conocimiento. Enrique Dussel. Un proyecto ético y político para América Latina donde detalló la cronología de los estudios y obras. También tiene una parte dedicada a los libros de y sobre Dussel pero, como es obvio, solo hasta la fecha indicada. Desde entonces, Dussel ha producido bastante, incluso, podríamos decir que sus obras de mayor sistematicidad. Otro trabajo interesante en esta línea, es el editado por Martín Alcoff y Mendieta (2000) que reúne varios trabajos sobre la obra de Dussel. Además, en la introducción se señalan unas etapas que queremos tener en cuenta, para periodizar la bio-bliografía. A. Estudiante en Mendoza (1961). B. La reconstrucción del pensamiento filosófico europeo (1961-1969). C. Origen de la Filosofía de la Liberación (1969-1976). D. Desarrollo de la Filosofía de la Liberación y el Exilio Mexicano (1976-1989). E. Nuevos Debates con Apel, Ricoeur, Rorty, Taylor y Vattimo (1989-1999). Estas etapas son para tener en cuenta en la breve cronología que haremos para ver la obra de Dussel en su desenvolvimiento. Ambos trabajos, el de Moreno (1998) y Martín Alcoff y Eduardo Mendieta (2000) cubren muy bien la producción teórica correspondiente al siglo XX.

La obra de Dussel en la actualidad la podemos abordar desde diferentes perspectivas, como por ejemplo, una lectura muy interesante sería desde Decolonial Turn (Maldonado-Torres, 2008: 187-236) que gana terreno en las discusiones de pensamiento crítico a nivel mundial. Lo otro, pero que también va en la misma dirección, es lo que se conoce como pensamiento crítico transmoderno (Bautista, 2014: 19-86). En ambos casos se transita por el mismo camino, una crítica radical al pensamiento moderno occidental y la condición de posibilidad de otro mundo posible. En ambos casos la localización, es decir el contexto "desde" donde se enuncia la crítica es muy importante para un pensador de la liberación:

"Localización" indica la acción hermenéutica por la que el observador se sitúa comprometidamente en un lugar socio-histórico, como sujeto de enunciación de un discurso, y por ello es el lugar desde donde se hacen las preguntas problemáticas [...] Esta actitud crítica de intentar tener el mayor grado posible de reflexividad sobre el lugar desde donde se enuncia el discurso deberá mantenerse como posición permanente. (Dussel, 2007: 15). 
El pensador crítico de la liberación está comprometido con el lugar "desde" donde lanza la crítica, no puede pensar abstractamente únicamente, sino, en lo próximo a la materialidad, a la corporalidad. La mayor parte de la obra de Dussel es una permanente propuesta crítica interdisciplinaria contra la dominación, producto de la modernidad occidental, o para ser más certero, decir como Walter Mignolo "The darker side".

Ahora veamos sucintamente la obra cronológicamente y su evolución. Dussel desde la década del cincuenta estudia el tema del bien común; tanto en su tesina de licenciatura en la Universidad Nacional de Cuyo La problemática del bien común en el pensar griego hasta Aristóteles (1957), como en su tesis doctoral en la Universidad Central de Madrid El bien común. Su inconsistencia teórica (1959), lo demuestran así. Esto es un primer momento en su formación académica donde se enfatiza el estudio de los clásicos.

Inicia sus estudios doctorales en Filosofía e Historia. En este periodo hay varias cosas que confluyen, su experiencia en Oriente le amplia el horizonte (el mundo semita) y escribe en 1969 El humanismo semita. Estructuras radicales del pueblo de Israel y otros semitas. Había publicado un poco antes, en 1967, Historia de la iglesia en América Latina. Coloniaje y liberación 1942/1973. En 1970 publica El episcopado latinoamericano. Institución misionera en defensa del indio (1504-1620) (9 tomos) que es su tesis doctoral en Historia en la Sorbona. También lee seriamente a los fenomenólogos europeos más importantes y descubre la obra de Emmanuel Lévinas. En 1967 retorna a Argentina definitivamente. En medio de este breve periodo nace lo que se conoce como el movimiento Filosofía de la Liberación. Desde finales del sesenta sus investigaciones en Europa y la realidad latinoamericana le abrieron una nueva línea de investigación: la Ética. Publica cinco tomos en 1973 Para una Ética de la Liberación Latinoamericana. En ese mismo año sufre un atentado de bomba en su residencia que lo obliga a salir de Argentina definitivamente, después intentó volver pero el esfuerzo quedó en vano.

Desde mediados de los setenta inicia su etapa como exiliado, profesor en México (Titular en la UAM desde 1975, y a partir de 1976 imparte cursos en la UNAM). Publica en 1977 su Filosofía de la Liberación. Estudia sistemáticamente a Marx, saca varios tomos al respecto, confrontando el marxismo ortodoxo con el heterodoxo (no es el primero), lo que permite tener una base material a sus posteriores trabajos. Este ciclo de trabajos los comienza con una obra interesante publicada en 1984 Filosofía de la producción, en la que no trata exclusivamente de Marx, pero que tiene todo un apartado sobre él, en particular sobre el "Cuaderno tecnológico - histórico (1851)" y también sobre su técnica de estudio, de anotar en cuadernillos, que llegó a compilar cientos de ellos. Culmina este período con la publicación en 1993 Las metáforas teológicas de Marx. Pasando por los textos en esta línea: en 1985 La producción teórica de Marx. Un comentario a los Grundrisse. En 1988 Hacia un Marx desconocido. Un comentario de los Manucritos del 61-63. Y, en 1990 El último Marx (1863-1882) 
y la liberación latinoamericana. Un comentario a la tercera y a la cuarta redacción de "El Capital".

A partir de 1985 se realizan las "Primeras Jornadas Germano-Iberoamericanos de Ética", y es a partir de 1989 que se encaran explícitamente dos éticas, de dos contextos diferentes, la Ética del Discurso de Apel y Habermas y la Ética de la Liberación de Enrique Dussel. Estas jornadas-encuentros fueron organizadas por Raúl Fornet-Betancourt (1992), este encaramiento fue una real puesta a prueba del diálogo intercultural. A este diálogo corresponde el texto publicado en 1992 Fundamentación de la ética y filosofía de la liberación. También hay un debate con Richard Rorty, con respuestas de Paul Ricoeur y Apel, a esto responde el texto publicado en 1993 Apel, Ricoeur, Rorty y la filosofía de la liberación con respuestas de Karl-Otto Apel y Paul Ricoeur. También en 1998 publica Ética de la liberación ante el desafío de Apel, Taylor y Vattimo con respuesta inédita de K.-O. Apel. A partir de este momento se consolida como uno de los pensadores latinoamericanos de reconocimiento mundial.

Continúa su crítica radical a la modernidad occidental que ya había iniciado con la sistematización de una institución (la iglesia) en Abya Yala, lo que le permitió la visión histórica a la vez que teológica de un arco de tiempo bastante extenso que permite ver variables. Con sus lecciones en Frankfurt publica en 19941492 El encubrimiento del otro. En 1998 después del largo debate con Apel puede ajustar cuentas con la Ética, diríamos que la retoma y la pone en un contexto contemporáneo y global, es decir, en el contexto de la globalización; a esto corresponde la publicación en 1998 de Ética de la Liberación en la edad de la globalización y de la exclusión.

En la primera década del presente siglo, inicia la publicación de varios artículos y conferencias sobre Política, cuestión que viene trabajando hasta la actualidad. Publica en 2001 Hacia una filosofía política crítica, en 200620 tesis de política. En 2007, en vísperas de publicar el primer tomo de Política de la liberación, publica Materiales para una política de la liberación. El material compilado estaba previsto que saliera antes que el primer tomo, pero son temas que se subsumen en los tratados sistemáticos de la Política. Todos estos trabajos son preparatorios, así como Marx escribió los Grundrisse antes de escribir El Capital. Para aclarar ideas y posibles hipótesis de trabajo, esta es una etapa madura de profunda reflexión. El producto de eso es en 2007 Política de la Liberación. Historia Mundial y Crítica. Y, en el 2009 Política de la Liberación. Volumen II Arquitectónica. La Política está pensada como una trilogía.

En el 2011 se publicó el enorme volumen El pensamiento filosófico latinoamericano, del Caribe y "latino" 1300-2000 de 1.110 páginas, editado junto a Carmen Bohórquez y Eduardo Mendieta. En 201416 tesis de economía política. Interpretación filosófica. Estas tesis se tienen que leer después de las 20 tesis de política, son complementarias. En este lapsus de tiempo hay algunas otras cuestiones menores pero que son subsumidas. Lo actual de su producción es reto- 
mar las viejas problemáticas pero con mayor vitalidad, las ha recopilado en el 2015 bajo el título Filosofías del Sur. Descolonización y Transmodernidad, y en 201614 tesis de ética. Hacia la esencia del pensamiento crítico. Si leemos estos textos bajo el denominado giro decolonial veremos lo enriquecedor que es el pensamiento de Dussel para los diálogos críticos tan necesarios en el siglo XXI para encarar la realidad.

\section{Algunas dificultades comunes en la recepción}

Después de revisar los planteamientos de Moreno, Alcoff y Mendieta y la producción teórica, tomaré en consideración algunas de las dificultades que identificó Juan José Bautista (2014) en su libro rojo, que son las más comunes en la recepción de la obra de Enrique Dussel. La primera, que también es central para toda su obra, está referida a lo que es constante en la Filosofía, la dificultad de comprender lo nuevo ante lo viejo o lo crítico ante lo tradicional.

Hay varios autores críticos de la modernidad, pero no en el sentido de darle un carácter totalizador a ésta. Dussel en un intento de hacer un pensamiento crítico de la modernidad occidental está problematizando todo. En cambio, hay muchas críticas, pero parciales. Esa es una de las razones por la cual un lector que se acerca a esta obra le resulta muy abarcadora, al punto de parecerle pretenciosa. Como es un pensamiento que sigue un programa, el lector que se adentre de forma parcial sin conocer el universo del autor se encontrará desperdigado. Otra cuestión en esta misma línea es que, como es un autor que hace una lectura interna de los clásicos y el canon que deconstruye todo lo leído para erigir su punto de vista, puede parecer trivial si no se tiene en cuenta lo antes dicho.

Lo enriquecedor es que la lectura de Dussel obliga al lector a contrastar las fuentes y sacar sus propias conclusiones. Al hacer una lectura desde dentro de los autores, pensando en los problemas objetivos, re-incorpora los conceptos a su programa bajo un nuevo signo, esto es lo que se conoce como el pensar categorial, darle un significado al concepto para su andamiaje teórico. Como es un pensamiento que parte de algo concreto -el dominado-, entonces es acusado de que no es Filosofía, y no pocas veces es acusado de ideológico.

Este pensamiento, al tener su propio núcleo de enunciar la crítica desde el Otro oprimido, es muy difícil que incorpore estos conceptos que no están registrados comúnmente en nuestra estructura mental, más que como alteridad. Dussel lo entiende y su crítica se enfoca en la proximidad del Otro. Hay que ser reiterativo en señalar que no se trata de una negación total del pensamiento de la modernidad occidental, sino, un "intento de lograr un nuevo criterio ético o principio epistemológico" (Bautista, 2014:25). Creo que este es el núcleo que dificulta a un lector adentrarse en la obra de este pensador que lo único que propone es la potencialidad del pensar desde aquel que la modernidad occidental encubrió. 
En otro segmento de dificultades también podemos decir que Dussel es un pensador intempestivo. Esto no quiere decir que no está al tanto de lo más reciente de la academia, todo lo contrario, está enterado prácticamente de todo. Está fuera de tiempo en el sentido en que va en contra de lo planteado, del "establishment", por ejemplo, cuando escribió su Ética no era una preocupación esta temática, cuando escribe sobre Marx tampoco y lo mismo sucede con la Política. Otro ejemplo más reciente es que desde finales del sesenta Dussel ya tenía un discurso decolonial, y ahora empieza a tomar fuerza a nivel mundial el Giro Decolonial (Cf. Maldonado-Torres, 2006, 2008).

Sobre el cómo pensar y articular una praxis, hay que tener en cuenta que son los movimientos sociales, el agitar de la gente y de los pueblos en disconformidad con el sistema, los que hacen surgir las reflexiones teóricas, y no al revés. Otra dificultad es que todo el pensamiento de Dussel se ha negado hacer de su pensamiento un asunto pragmático, más bien optó por mantenerse desde su lugar de enunciación y erigir su crítica desde (con) el oprimido, y como la Teoría Crítica en sus momentos, sin negar o dar como anticuada la tradición hegeliana-marxista.

Esto deja en desventaja a toda la Filosofía de la Liberación, dado que el pensamiento en su mayoría se ha convertido al pragmatismo. En cierto sentido es una renuncia al pensamiento dialéctico. Es que vivimos en tiempos inmediatos que requieren respuestas inmediatas y qué mejor que el pragmatismo. Entonces, el pensamiento de Dussel es una pretensión de superación del pensamiento moderno occidental abriéndose paso en medio de este.

\section{Pensamiento crítico de la liberación}

El pensamiento crítico de la liberación de Dussel es una crítica radical al pensamiento moderno occidental. El pensamiento moderno occidental es el canónico, es el que hay que leer, es la regla para el pensamiento universal, se le puede leer en los siguientes sentidos: para imitarlo, superarlo, rechazarlo o incluso ser indiferente. En el caso de Dussel podríamos decir que su objetivo es subsumirlo. En la Filosofía lo más frecuente es lo primero, ya que es muy difícil salirse de los márgenes de lo moderno occidental, así que lo más frecuente es la imitación, es decir pensar como los filósofos modernos europeos, a pesar de que a veces los esquemas estén pensados desde un determinado contexto y no funcionen en otro. Es muy difícil salirse de ese canon, ya que es lo habitual, las costumbres tienen un gran peso sobre la psique de la humanidad, lo cual condiciona el actuar y el pensar.

Solo hasta el siglo XX podemos ver que hay un afán de hacer lo propio desde Latinoamérica, y no solo imitar. En la literatura lo que se conoce como el boom latinoamericano, que es un giño de originalidad, expresó con su propia dinámica lo que era Latinoamérica. En la actualidad la indiferencia a lo propio (hasta un Cicerón reivindicaba lo propio ante lo griego) se debe más que todo a la ignorancia. Una opción de leer el pensamiento moderno occidental es intentar 
superarlo, esto es lo agonístico, lo que necesita ser subsumido. Creo que a esto último apunta Dussel, de una forma radical, no como una negación de lo moderno, sino como intento de superación.

En la presentación de Filosofía de la liberación latinoamericana de Cerutti (2006:34), Leopoldo Zea señaló que una de las vertientes de la Filosofía de la Liberación estaba encabezada por Dussel, y buscaba: "destruir la filosofía europea occidental para iniciar en esa América una filosofía que habrá de ser original desplazando a la primera." Hay otros elementos interesantes en esta introducción, por ejemplo, la dependencia del lenguaje "alambicado" propio de los fenomenologos europeos al cual recurre Dussel. Este herencia es muy dificil de quitar.

Veamos de qué trata este destruir. Esta destrucción lo es como edificación de algo nuevo. Para eso era necesario elaborar un programa amplio que brindara las herramientas para esa "destrucción" de la Historia. Para ello es imperante un nuevo relato histórico mundial. Ya en 1965 Dussel publicaba en Revista de Occidente:

La Historia se ha transformado en el medio privilegiado de formar y conformar la conciencia nacional. Los gobiernos, las élites dirigentes, tienen especial empeño en educar al pueblo según su modo de ver la Historia. Esta se transforma en el instrumento político que llega hasta la propia conciencia cultural de la masa y aún de la "Inteligencia". Los que poseen el poder, entonces, tienen especial cuidado de que la periodificación del acontecer histórico nacional sea realizada de tal grado que justifique el ejercicio del gobierno por el grupo presente como un cierto clímax o plenitud de un período que ellos realizan, conservan o pretenden cambiar. (Dussel, 1966:12).

La Historia es central para dicho programa, así veremos que para darle un giro a la instrumentalización que se dio, habrá que crear una conciencia histórica (la memoria histórica que tiene incidencia en el imaginario, y que se materializa en la acción del sujeto), partiendo de una nueva comprensión de los hechos históricos (los hechos no varían, las interpretaciones sí). Entonces se hará imperante la destrucción de la Historia. Para tener un nuevo relato donde asentar esa Filosofía de la Liberación, es decir una filosofía que tenga como horizonte la liberación, la del sujeto (ser humano) y la del pueblo (la humanidad). Esto sería otro gran relato que criticarán los posmodernos. Habría que recordar que Dussel es uno de los primeros en utilizar el concepto "filosofía posmoderna" en las palabras preliminares de su Filosofía de la Liberación publicada en 1977. Es decir, dos años antes que Jean-François Lyotard escribiera La condición posmoderna.

La de-strucción se da en todo el pensamiento de Dussel, donde la Historia es el eje rector. Ya lo decía en uno de sus primeros trabajos de Ética: "La «de-struc- 
ción» de la historia de la ética no tiene un mero carácter destructivo sino crítico." (Dussel, 1973:5). En su Filosofía de la Liberación también diría que "se inicia lentamente una des-trucción [...] de la historia." (Dussel, 2011:17). En la Política lo hace de forma más sistemática, en cuanto que intenta de manera parcial-introductoria "exponer una posible historia de la política." (Dussel, 2007b). No es una especie de historia universal, tal cual, sino un relato desde donde potenciar su pensamiento crítico, o desde donde impulsarse para superar los siguientes limites: helenocentrismo, occidentalismo, eurocentrismo, colonialismo mental y teórico, y por último el no incluir a América Latina en la Modernidad. Esto es lo que podríamos llamar el proyecto de crítica radical al pensamiento moderno occidental.

Hay que tener en cuenta que los primeros trabajos de Dussel son de Historia y Teología. Incluso podemos afirmar que es a partir de estos que se puede erigir su programa filosófico. Podemos plantearlo en el siguiente orden interrelacional: Ética, Filosofía, Política (aún falta el tercer tomo) y una Económica en clave de la liberación. Que siempre están interconectándose. Este es un programa en curso, aún no definitivo, ya que se encuentra en estos momentos trabajando en una lógica de la analogía-liberación.

\section{Dominación-liberación}

Podríamos decir que las reflexiones de Dussel tienen como punto de partida un marco histórico-teológico (sus primeros trabajos fueron sobre los semitas y la historia de la Iglesia) que tiene como nodo la dicotomía dominación-liberación. Muchas veces se le endilga a Dussel el calificativo de teólogo, como algo despectivo. Cabe recordar que Marx en su tesis doctoral señaló algo muy interesante al respecto que a veces solemos olvidar, es la existencia de "el vinculo entre el entendimiento teologizante y la filosofía." (Marx, 1971: 10). De ahí la relación intrínseca entre la Filosofía y Teología de la Liberación que se dio a finales de los sesenta. Nacieron en el mismo contexto, y en particular en Dussel con vínculos muy estrechos.

La cuestión dominación-liberación es el nodo de donde van a salir las diversas ramificaciones del pensamiento crítico de la liberación de Dussel. Esta paradoja dominación-liberación es central (van unidas por un guion, tienen una relación cercara y comprometida) y hay que comprenderlas en ese sentido; si fueran divergentes, se hubiese puesto en vez del guion una proposición particular negativa (o). O si fuera una relación difusa o sin compromiso, se hubiese puesto una conjunción copulativa (y). Pero, como éste es un pensamiento -no solo de-, sino también comprometido con la liberación, entonces analiza esta paradoja como relacional y con compromiso.

En la primera parte del capítulo I, del texto Historia de la iglesia en América Latina. Coloniaje y liberación, Dussel (1974) se trata esta cuestión. Libro escrito en la década de los sesenta, es poco citado. Cuando se tratan estos temas, se va 
directo a la Ética (Dussel, 2014a) o a la Filosofía (Dussel, 2011), por lo que pasa desapercibido este texto que es el leitmotiv de toda la crítica posterior. En el apartado de dominación-liberación Dussel ya se plantea el carácter extra regional de este pensamiento, que "no es sólo válido para nuestro continente sociocultural, sino para todas las culturas periféricas" (Dussel, 1974a: 25) (el subrayado es nuestro). Esto va ser muy recurrente en toda la Filosofía de la Liberación en su conjunto, posteriormente dará paso a un real diálogo global de saberes, que ya se está viendo en la práctica (Cf. Dussel, 2015: 11-30).

La paradoja en cuestión se manifiesta en lo que se vivió en el encuentro de dos culturas divergentes; la de los pueblos aurorales de Abya Yala y los de Occidente. La dominación que se impuso a partir de 1492 contra el africano (antes), el indígena, a escala mundial dio forma al nuevo moderno sistema mundial (diría Wallerstein) que tensaba la paradoja. Al mismo tiempo que se quiere dominar al indígena que estaba allí, al negro traído de África para ser esclavo, también se quiere lo contrario, la liberación. Esta paradoja entonces nos plantea el punto de partida. A partir del siglo XVI la humanidad pudo entrar en contacto casi en su totalidad.

El tema central de la paradoja es la injusticia a partir del encubrimiento en 1492, en tres niveles que acompañarán toda la obra dusseliana, que mientras más se avance en la lectura de las obras maduras se verán con mayor claridad. Y que en el camino también irán evolucionando.

En pleno siglo XVI, en 1552, Bartolomé de las Casas acusa a los cristianos europeos de injusticia, porque a «los hombres varones (ya que comúnmente no dejan en las guerras a vida sino los niños y mujeres) se les oprime con la más dura, horrible y áspera servidumbre». La posición hermano-hermano (varón, oprimido, débil) es el nivel político; la posición varón-mujer (casa, esposa, viuda) es el nivel erótico; la posición padreshijos (huérfano-niños) es el nivel pedagógico. (Dussel, 1974a: 26).

Esta nueva organización del mundo a partir del encuentro ya no se basa en que los humanos son una especie de semejantes entre sí, sino, que remarca las desigualdades de razas, en superiores e inferiores. Esto era nuevo en el sentido que se hace mundial, porque ya se había puesto en práctica cuando los griegos consideraban a los demás barbaros y los romanos colonizaban gran parte del Mediterráneo, y no todos eran ciudadanos. La novedad con el control del Nuevo Mundo consistía en que la cuestión era mundial. "El yo conquisto al indio" era crucial para ello, y la centralidad de lo que hoy es Europa también. El indio conquistado que tiene unas tierras productivas, posee metales preciosos y puede ser explotado por el que es superior.

La indígena es violada por el español, la libido lo impulsa a tener sexo, pero a su esposa en el hogar le pide castidad, la indígena desnuda ante el violador sufre las vejaciones. Entonces, el "yo conquisto", que es el varón conquistador, 
domina al indio sui géneris políticamente y eróticamente a la indígena. El tercer nivel corresponde a lo pedagógico; el conquistador que vence con las armas y atraviesa los cuerpos con su espada también proyecta en el indígena su civilización, su cultura, pero éste en condición de inferior por su raza reproduce la cultura de su amo.

\section{Aspectos básicos de la Ética de la Liberación}

Para una cuestión metodológica hay que comprender la Ética como introducción al tratado de Política. Como dijo Bernardo de Chartres "somos como enanos a hombros de gigantes." Así, para la Política hay que subirse sobre Aristóteles, y en cuanto a la liberación sobre Dussel (y los filósofos de la liberación). Vamos a tomar un ejemplo canónico para comprender el orden que utilizó Dussel en su programa en cuanto a la Ética y la Política. Aristóteles primero escribió las Éticas (Magna Moralia, Eudemia y Nicomáquea) y luego la Política; como señaló García (1988), la Ética es como una introducción a las cuestiones políticas, estas hay que entenderlas como ámbitos del saber práctico. Utilizamos a Aristóteles como ejemplo porque la tradición no se puede deshacer de un plumazo, está allí como base. Aristóteles en su Ética a Nicómaco señala:

Parece que toda arte y toda investigación, e igualmente toda actividad y elección, tienden a un determinado bien; de ahí que algunos hayan manifestado con razón que el bien es aquello a lo que todas las cosas aspiran. Aunque es claro que existe una diferencia entre los fines: en efecto, en unos casos hay actividades, mientras que en otros hay ciertas realizaciones que acompañan a estas. (Ética, Libro I, §1, 1094a).

Cada actividad humana tiene un fin determinado y este fin tendría que suponer un bien, pero esto no se da por sí sólo; para Aristóteles se da por medio de la virtud. Al tratarse de las cuestiones humanas, hay que pensar rigurosamente para actuar correctamente. Parece que la naturaleza humana no necesariamente transita por los caminos del "bien", más bien hoy la humanidad desciende hacia la barbarie. Es decir, es necesario saber a qué fin queremos Ilegar.

Si ello es así, habrá que intentar captar, al menos mediante un bosquejo, cuál es este fin y a cuál de las ciencias o facultades pertenece. Pareciera que pertenece a la más importante y a la directiva por excelencia, y es manifiesto que ésta es la Política. (Ética, Libro I, §II, 1094b).

Utilizamos aquí a Aristóteles para ver el programa de Dussel en referencia a la Ética y la Política, teniendo en cuenta que entre la una y la otra Dussel escribió varias cuestiones. Este es el peso de la tradición, de la que no se debe renegar, sino criticarla. Si con Aristóteles decimos que hay que practicar la virtud, con Dussel tenemos que decir que tenemos una práctica de la liberación. Recientemente Giorgio Agamben señaló: "No creo que en filosofía se pueda 
distinguir, como se hace en la Universidad, entre filosofía de la política, de la moral, del lenguaje. La filosofía es única. La filosofía es siempre política" (cf. Arroyo, 2016).

En un primer momento, podemos ver que Dussel se plantee en Para una Ética de la Liberación (tomo I y II) la fundamentación ontológica del Otro, y la praxis liberadora ante la totalidad, por su influencia de los fenomenólogos franceses. En un segundo momento confrontándola con el giro pragmático de la Ética del discurso de Apel, entonces se puede producir una Ética de la Liberación (1998) (la amarilla como diría Juan José Bautista) que parte del principio material, la vida. Igual que Franz Hinkelammert.

En la tesis 3 del apéndice I lo define de la siguiente manera:

La vida de que hablamos es la vida humana. Por humana entenderemos la vida del ser humano en su nivel físico-biológico, histórico-cultural, ético-estético, y aun místico-espiritual, siempre en un ámbito comunitario, la vida humana de la que hablamos no es un concepto, una idea, un horizonte ontológico abstracto o concreto... La vida humana es un modo de realidad, es la vida concreta de cada ser humano desde donde se encara la realidad (Dussel, 1998b: 618).

Sólo partiendo desde este principio material se pudo encarar el giro pragmático, de lo contrario la Ética de la liberación hubiese quedado subsumida por la Ética del discurso, peor aún, este principio material quedaría en un segundo plano ante la comunidad ideal, cuando la vida humana debería ser el eje rector de toda la fundamentación de la Ética. Solo esta confrontación amical ayudó a re-formularse sus planteamientos éticos. Gracias a este encuentro la obra de Dussel empieza a tener un impacto mundial, ya que se materializa y se demuestra empíricamente la posibilidad de un diálogo intercultural (Sur-Norte). A tal punto que Dussel le dedicará la Ética amarilla a Apel ("A Karl-Otto Apel, que se atrevió a dialogar con filósofos del Sur inspirándonos teóricamente").

Aspectos básicos de la Filosofía de la Liberación

¿Por qué poner a la Filosofía en medio de la Ética y la Política? Hay razones cronológicas y de orden programático. En ambos casos, tanto en la Ética como en la Política, Dussel les da un tratamiento desde la Filosofía. Pero, como ya hemos visto, hay una relación intrínseca entre estos ámbitos del saber; en filósofos europeos, como Aristóteles y Agamben, no nos cabe la menor duda de que Dussel siempre está en diálogo crítico con la tradición. Desde el punto de vista cronológico, primero escribe Para una Ética de la Liberación (varios tomos), al tiempo que emerge en Argentina lo que se conoce como la Filosofía de la Liberación; en la segunda mitad del setenta escribirá una obra con ese mismo título. Más adelante retomará los temas éticos, escribe entonces una Ética de la 
Liberación, pero se da cuenta que su Filosofía no puede ser una Ética, y siguiendo a Aristóteles escribe una Política en curso.

Después de la disputa entre Leopoldo Zea y Augusto Salazar Bondy, el esfuerzo más denotado que ha tenido el pensamiento filosófico latinoamericano después de la segunda mitad del siglo XX y lo que lleva de este, es la Filosofía de la Liberación (Cf. Cerutti, 2006). Incluso podemos decir que la Filosofía de la Liberación es el resultado de aquella disputa. Pese a la divergencia de este heterogéneo movimiento intelectual, su unidad es la liberación, la liberación desde Latinoamérica, con la salvedad de que esta preocupación filosófica ya traspasó las barreras regionales, es mundial. La Filosofía de la Liberación ha subsumido diversas e interesantes propuestas, en particular, la Teoría de la dependencia y la Teología de la liberación, aplicándola en los nuevos escenarios del siglo XXI.

Sobre la Filosofía de la Liberación hay dos cuestiones que resaltar. "Será esta corriente la más original y representativa de la producción filosófica latinoamericana" [...] Y que trata de una "confrontación radical con el pensamiento europeo-occidental." (Beorlegui, 2010:661). Por un lado está lo que podríamos denominar crítica radical al pensamiento moderno occidental, que es lo representativo, a esto lo han Ilamado el giro decolonial (Cf. Maldonado-Torres, 2006, 2008). Y lo segundo es lo que se conoce como el diálogo intercultural (Cf. Fornet-Betancourt, 2004). Estas dos cuestiones son de suma actualidad. Esto es lo sustantivo en esta Filosofía que abre nuevos caminos para el diálogo mundial de pensamiento crítico.

Para conocer los aspectos básicos de la Filosofía de la Liberación habría que ubicarla en un contexto más global, en el del pensamiento posmoderno. El texto Filosofía de la liberación de 1977 de Enrique Dussel es una referencia obligatoria para ello. El texto citado está enmarcado en la corriente de la "posmodernidad" que trajo consigo un gran revuelo en la cultura occidental, donde grosso modo el análisis consistía en describir el estado actual de la cultura desde todos los ámbitos después de las grandes transformaciones del capitalismo; el conocimiento dentro de estas grandes transformaciones no solo produjo un saber legitimador, sino instrumental para esos fines. Cuando Lyotard en La condición posmoderna (1979) describe esto de forma contundente y se hace popular la posmodernidad, ya Enrique Dussel y los filósofos de la liberación a finales del sesenta estaban desarrollando un pensamiento en esa dirección, en ese momento "desde" Latinoamérica. En particular Dussel había escrito en 1977 su Filosofía de la liberación, texto que es de lectura obligatoria para todo aquel interesado en el pensamiento filosófico de la liberación latinoamericano, en vista de que allí están sintetizadas muchas ideas tratadas de forma más densa en las obras anteriores y posteriores a esta; es un libro que puede servir como punto de encuentro. El texto en cuestión está escrito en breves aforismos en forma de sentencias. Las diversas traducciones muestran el gran impacto de la obra más allá del ámbito local-regional. Definitivamente la obra en cuestión ayudó a 
formular una nueva visión en la filosofía, como se lo planteó Lyotard después: la crítica a los metarelatos. La Filosofía de la Liberación era en particular la crítica al gran relato de la filosofía eurocéntrica, que ahora tendrá que ser criticada y auto criticada para no ser más de lo mismo y no convertirse en lo que criticó.

\section{Aspectos básicos de la Política de la Liberación}

Ahora vamos a tratar de explicitar -en sus elementos básicos, lo que le da contenido- a la Política en el programa filosófico de Dussel. En el primer tomo de su Política de la liberación esta filosofía política moderna, que es considerada universal, en verdad responde a necesidades objetivas de un contexto específico, y no al universo. Además es dominante, posicionándose por encima de los otros conocimientos sobre diversas formas de organización humana. Para Dussel esta intensión universalista tiene varios límites que habrá que superar desde el horizonte de la liberación, al menos desde los pueblos periféricos, desde los países oprimidos-dominados-dependientes. Hay que situar a la proyectada Política de la liberación con sus antecedentes, esta Política es posterior a la Ética. La Política está antecedida por varios textos (producto de seminarios, conferencias, artículos de periódicos, debates y clases) que van formando varios compendios, antologías y libros. Según su orden de aparición Hacia una filosofía política crítica, 20 tesis de política, y por último Materiales para una política de la liberación, serían entonces una transición de la Ética a la Política y también un paliativo para la formulación de las principales tesis expuestas en los siguientes tomos de la Política de la liberación: el tomo I Historia mundial y crítica y el tomo II La arquitectónica. En todos estos textos hay un constante diálogo con los principales pensadores contemporáneos, para mencionar algunos: Amartya Sen, John Holloway, Atilio Boron, Ernesto Laclau, Agnes Heller, Martin Jay, Immanuel Wallerstein, Judith Butler y otros. Como hemos dicho, hay decenas de trabajos fragmentarios que anteceden la Política de la liberación, pero que son subsumidas por ésta.

Entrando en la materia de la Política, muchos de los problemas que enfrenta la humanidad hoy son producto de deliberaciones políticas, de los llamados profesionales de la política que no piensan en la "vida humana en comunidad", sino que ponen de imperativo otros factores ( $p$, e. el financiero). Continentes enteros fueron y son devastados por la vorágine del capitalismo, y luego "la macro-política", las deliberaciones políticas de los (grandes) Estados desarrollados, le dan la espalda y sólo vuelven la mirada cuando estas regiones (afectadas) son funcionales para los intereses que ellos consideran rentables. Por más de cinco siglos los grandes Estados (desarrollados) de hoy, usurparon las riquezas de Latinoamérica y África (por citar dos ejemplos), ahora le cierran sus puertas a miles de africanos que buscan refugiarse en los países europeos o en Estados Unidos, cientos de estos mueren en el Mediterráneo. También surge una cierta empatía instrumental con Latinoamérica cuando ésta nuevamente empieza a figurar (en informes del Banco Mundial) como despensa mundial de ali- 
mentos en una eventual crisis alimentaria. Esto es otro falso dilema porque el problema del hambre no es la falta de comida, sino el desperdicio.

Con un grado de urgencia es necesaria "la participación pública, efectiva, libre y simétrica de los afectados" más allá de la comunidad ideal de hablantes, que es irrealizable en las condiciones materiales asimétricas donde se mueven los que representan (es decir los políticos) con los representados, víctimas de un sistema (totalizador) excluyente. En ese sentido el sujeto que vive en comunidad tiene que exigir y participar activamente, ya no sólo como representado, como en la democracia representativa, sino como actor de su propio devenir democrático. Para conseguir lo que le da sentido a la política, que es la "producción, reproducción y desarrollo de la vida humana en comunidad", es necesaria la participación para la toma de decisiones colectivas; esto es muy serio para dejárselo a los políticos. Las víctimas de un sistema mundial excluyente, no son pocas, son miles, millones en diferentes latitudes y diferentes grados de afección, tienen que pensarse como individuo autónomo, no individualista (el neoliberalismo impulsa el individualismo). Tiene que pensarse en comunidad y construir una forma de gobierno más participativa, donde democráticamentelegítimamente las mayorías de los afectados del sistema puedan tomar sus propias decisiones en conjunto. La práctica de la liberación es anterior a cualquier teorización de la liberación.

Los nuevos cimientos para otra visión -no eurocéntrica- del pensamiento político o historia de la política, tienen que construir sus bases a partir de la superación de los límites de esa visión. Diría Immanuel Wallerstein que las ciencias sociales están obligadas a superar el eurocentrismo (Cf. Wallerstein, 2000). De lo que se trata (como objetivo) es de "exponer una posible historia de la política" (Dussel, 2007b:11), una historia realmente crítica y mundial, que exprese un verdadero pensamiento político en su totalidad y no uno fragmentado. En la visión eurocentrica (que toma como modelos universales a la cultura griega y romana unicamente) los otros pueblos y culturas son ignorados o relegados a un segundo plano (en algunos casos, su forma de organización política fue aniquilada).

Esto no quiere decir que en el mismo centro de esta visión no exista autocrítica. Esto nos obliga a hacer una lectura cuidadosamente crítica. Reitero nuevamente que no se trata de una negación total de lo moderno europeo. Lo eurocéntrico es cuando Europa se toma a sí misma como centro (y en este caso Norteamérica es una extensión en otro continente). No por leer a los europeos se es eurocéntrico, eso es un argumento infantil. Incluso el propio Dussel parte de los europeos, por ejemplo de "la destrucción ontológica (Heidegger), deconstrucción discursiva (Derrida), reconstrucción epistemológica (Habermas) [.] desde los vencidos, desde las víctimas de la modernidad." (Dussel, 2007:15-16). Creo que esto último, el subrayado es nuestro, es la clave para entender la Política de la Liberación. Como va evolucionando, en su última obra 14 tesis de Ética, dirá que hay un aspecto correctivo con respecto a la posición de Heidegger 
que ahora la crítica, dado que el pensamiento de Heidegger, según Dussel, fue funcional a la moral del sistema vigente, es decir, del Führer.

Otro tema recurrente en la Política es el pensar estratégicamente. Dussel siempre esboza una estrategia, donde aclara su posición (localización) con respecto a las demás y desde allí enuncia su crítica (discurso). Podríamos decir que la estrategia consiste prima facie en una ruptura con la (vieja) visión y la construcción de una (nueva) visión realmente crítica y mundial de la Política, donde se dé cabida a una ampliación de la participación política, es decir, que no queden excluidos los pueblos aurorales de Abya Yala y del sur global (como diría Boaventura de Sousa Santos y María Paula Meneses). La crítica a este nivel se hace radical, cuando con el desarrollo de las ciencias sociales (como la antropología, la arqueología, la etnología) se descubren estas otras formas de organización, formas de pensar, formas diferentes de concebir el cosmos, se vuelve insostenible el eurocentrismo, pero aún no es suficiente cuando todavía se ningunea esas otras formas por estar en la periferia del centro desde donde supuestamente emergen ideas y formas organizativas políticas "autenticas" que se implantarían como modelos universales (lo políticamente correcto).

\section{Temas y debates en curso}

Es necesario afinar algunas ideas en torno al pensamiento de Enrique Dussel. Ya hemos visto de forma muy sucinta el nodo crítico y las partes elementales. Ahora podemos ver dónde estamos y para dónde queremos ir. Es un ir a contrapelo de lo establecido. De lo contrario se muere el pensamiento. Hoy estamos ante una coyuntura mundial -como diría Ulrick Beck- de riesgo, que desborda todo el planeta: la desigualdad Ilega a un porcentaje que raya en la obscenidad. La crisis migratoria a nivel global sigue aumentando, ya no solo en Turquía, también hay cientos de cubanos y africanos de paso por Panamá. Estos problemas se mundializan y las soluciones cada vez más son peores que el mismo problema en sí. En nuestra región, Latinoamérica, se desestabilizan los gobiernos, en particular los posneoliberales. En este estado de cosas se hace imperante una reflexión filosófica de la liberación, de la mente, de los seres humanos, de los pueblos oprimidos del mundo. Este es un esfuerzo que trasciende a esta generación que criticó la fundamentación contemporánea de la modernidad eurocéntrica.

La Filosofía de la Liberación tiene varios retos en el siglo XXI, primero soportar la crítica y sobrevivir en tiempos de adjetivación del pensamiento. El movimiento intelectual que conocemos con tal nombre no es homogéneo, hay sectores en su interior. El eje rector pese a las discrepancias es la liberación. Desde el primer manifiesto (1973) se tiene plena conciencia de que se parte "desde" el oprimido, el marginado, el pobre; "desde" la exterioridad del Otro. Esto quedó reafirmado años después (con el Manifiesto de Rio Cuarto) contra la vorágine del neoliberalismo, contra la especulación financiera que diezma la dignidad humana. Lo que impera en este pensamiento, son los cambios socie- 
tarios necesarios para otro mundo posible, donde la potencialidad de la vida humana sea la fundamentación. Hace poco el pensador boliviano Juan José Bautista se ganó el Premio Libertador al Pensamiento Crítico, a lo cual la colega Katya Colmeliza dijo “¡Feliz, la filosofía de la liberación continua!” Esta expresión es alentadora.

\section{Un proyecto para nuevas generaciones}

El pensamiento crítico de la liberación tiene que ser una empresa colectiva en cuanto a debates, producción de conocimiento, diálogos e intercambios, tiene que estar en movimiento, no se puede permitir estar en un estado de pasividad, sino en potencia. La investigación y la mística de la relación del maestro y el discípulo o la maestra y la discípula (o mixto) se diluyen en la realidad tecnocrática, la burocracia de las universidades está acabando con el conocimiento. La producción de conocimiento, el preparar la tesis se ha convertido en un proceso administrativo más que en la Bildung. Diríamos que esto es una de las principales limitaciones para las nuevas generaciones, siendo estas a veces los mayores culpables con su actitud apática.

En su Política Dussel desarrolla de forma más sistemática su pensamiento crítico de la liberación. En la misma dice que solo se está planteando hipótesis de trabajo, que deberán seguir otros/as, ya que es "un proyecto para varias generaciones por venir." (Dussel, 2007b: 11). Un proyecto que tiene que seguir, superar las limitaciones existentes, y recuperar la mística perdida y no truncarse por lo difícil del mismo. Los compañeros de viaje son pocos y hay que escogerlos muy bien, a esto me refiero con la empresa colectiva. Puede que el pensar en sí sea más bien una actividad un poco solitaria, pero la experiencia contextual del pensar tiene que ser colectiva. Las nuevas generaciones tienen la tarea de continuar con las hipótesis planteadas, una de las más interesante es la de investigar en clave decolonial, que es más enriquecedor que hacerlo pasivamenteacríticamente bajo el esquema colonial eurocéntrico.

\section{Filosofías del Sur}

Para ver un poco la perspectiva que se tiene del Sur, podemos observar en la literatura europea algunas referencia en cuanto la contraposición del Norte contra el Sur. Esto no es solo una cuestión de los latinoamericanos o los indios, sino que dentro de la propia Europa se puede ver la diferencia, un ejemplo de eso es el libro de Zizek y Horvat (2014) El sur pide la palabra. Ahí contrampone una Europa del Norte, de la especulación financiera, con una del Sur, que reivindica la justicia social y la participación ciudadana, éste más bien desde la resistencia.

Otra referencia del Sur es la de Dieter Richter, que nos da un pincelazo de cómo lo ve: 
El Sur. La historia de este punto cardinal es la historia del gradual descubrimiento de unos mundos extraños, tanto en los mapas geográficos como en los mapas del alma... La situada al sur fue durante mucho tiempo terra incognita, región tenebrosa de un hemisferio hostil a la vida. Poco a poco, buscadores de Dios, aventureros y descubridores fueron abriendo caminos hacia esas tinieblas (Richter, 2011:13).

Volvamos a Dussel en una de sus últimas obras Filosofías del Sur. Emulando -en el sentido del título- a Boaventura de Sousa Santos que primero publicó Una epistemología del sur y luego junto a María Paula Meneses Epistemologías del sur (en plural), trata la cuestión del Sur, entre otras cosas. Esto para dar cuenta de otro pensamiento, no sólo el que se produce canónicamente en Europa y Norteamérica, sino en la periferia global. Nótese que en ambos casos se plantean la cuestión en plural, es decir, no una epistemología ni una filosofía, sino varias, es la pluriversidad. La diversidad siempre enriquece los diálogos y los debates de cara a la complejidad de nuestro tiempo. En Filosofías del Sur Dussel se plantea la serie de debates del siglo XXI con el Giro Decolonial, con los Estudios Poscoloniales, la Teoría Crítica y los pensamientos críticos más relevantes. Estos debates tienen que mirarse desde la diversidad, de lo contrario poco o nada se entenderá. Una de las perspectivas más interesantes de estos últimos años es la que se conoce como el Giro Decolonial que empieza a ganar fuerza y formar parte del lenguaje y las investigaciones científicas, que vuelven a retomar cuestiones que de alguna u otra forma se quedaron en el camino. Nuevamente se problematiza las formas de colonialismo (p. ej. el colonialismo interno de Rodolfo Stavenhagen y Pablo González Casanova) y sus nuevas variantes. Esto no ha dejado de despertar críticas interesantísimas.

Michael Hardt ha escrito sobre la cuestión decolonial. Es muy interesante tener interlocutores que problematicen la cuestión. Él señala que uno de los problemas es cómo articular todas estas luchas por medio de lo común. Hardt junto a Negri son los teóricos de la multitud, y el pensamiento crítico de ambos consiste en pensar y re-pensar lo común. A partir de esto, Hardt plantea que hay ciertos límites del proyecto de-colonial, por lo cual, voy a copiar una larga cita, para saber cómo mira la cuestión decolonial:

Hoy surge un límite del proyecto de-colonial. Tengo dos ejemplos al respecto; el primero está relacionado con el sentido de la simple inversión, es decir, de la misma manera en que antes no era verdad que toda la potencia revolucionaria se concentraba solamente en el Norte, hoy tampoco es cierto lo inverso: que toda potencia revolucionaria se concentre únicamente en el Sur. El segundo ejemplo está vinculado al límite del pensamiento local, que pone un rompecabezas entre lo local y lo global, es decir ¿cómo pensar movimientos, estructuras y proyectos globales sin caer en la "unificación" o en el pensamiento único? Esto me parece un bloqueo al pensamiento local en cuanto tal. Estamos entrando en otra época en la que continúan las luchas 
contra el eurocentrismo, continua la lucha por la de-colonización, pero de otra manera. Estas luchas están caracterizadas por dos aspectos muy abstractos de esta nueva época. El primer aspecto consiste en el paralelismo de luchas. Claro que las luchas en diversos países de la sociedad son distintas por ejemplo, luchas feministas, antirracistas, de clase, de los pobres, etc. , pero que avanzan por caminos paralelos, es decir, son al mismo tiempo locales y globales. Entonces, ¿cómo se comunican estas luchas paralelas? [...] Con relación al segundo aspecto de esta nueva época de luchas contra el eurocentrismo, existía en la época anterior una contradicción filosófica entre diferencia e identidad, una pareja central del pensamiento contradictorio. Pero creo que las luchas hoy, ponen otra pareja de conceptos complementarios: la singularidad y lo común. Es decir, las luchas que están emergiendo hoy, no ponen el acento entre la identidad y la diferencia como base, pero sí en la singularidad y lo común. Estos son conceptos centrales en la noción de multitud. Esta pequeña introducción tenía dos objetivos. El primero quería situar el concepto de multitud como continuación del proceso de-colonial, pero subrayando su distinción. En lugar de hablar de una inversión del pensamiento local, su objetivo es desarrollar los caminos paralelos de las luchas. Segundo, en lugar de afirmaciones sobre la identidad y la diferencia, desarrollar la dinámica entre las nociones de lo singular y lo común. Hasta aquí he hablado en términos bastante filosóficos y abstractos; ahora voy a entrar en cosas más prácticas y simples. (Hardt, 2010: 67-67). (Las cursivas son nuestras).

Hardt es muy claro en ubicar el problema, en que uno de los fines de las diversas luchas se tiene que hacer desde lo común pero también teniendo en cuenta la singularidad de cada espacio, ese es el talón de Aquiles de las organizaciones que no saben articular la particularidad de movimientos con lo que los hace común. Así, por un lado las discusiones en las organizaciones sociales son interminables sobre los espacios que debe tener la singularidad de cada grupo, o por otro, se impone la línea ortodoxa de que solo la cuestión de clase o sindical (por ejemplo) es la verdadera contradicción, que las demás (las feministas, los inmigrantes, los gays, las lesbianas, etc...) no cuentan de mucho en la lucha. Este dilema aún no lo superan las organizaciones sociales. La propuesta de Hardt es pensar lo decolonial a partir de lo común. Pero no profundiza que en la perspectiva decolonial lo fundamental, a parte de lo que señalan tanto él como Negri, es des-montar la razón colonial desde su propio origen, al menos desde la formación del moderno sistema mundial capitalista.

\section{El paradigma de la liberación}

En su última obra -14 tesis de ética. Hacia la esencia del pensamiento crítiCO- Enrique Dussel (2016) incluye un epílogo un tanto ambicioso bajo el título El paradigma de la liberación. El concepto de paradigma nos remite obligatoriamente a la obra de Thomas Kuhn Estructura de las revoluciones científicas, en la 
cual el concepto paradigma tiene una singular importancia. Pese a que, desde una visión dusseliana se problematicen conceptos como universal, no podemos obviarlo cuando se habla de paradigmas en tanto que "realizaciones científicas universalmente reconocidas". En ese sentido, el pensamiento crítico de la liberación en Dussel es mundialmente, en vez de universalmente, más que reconocido, problematizado. Bajo esa lupa se podría ver el paradigma de la liberación, incluso como un movimiento más allá del autor.

Este último libro plantea temas sugerentes en la medida que le hayamos dado seguimiento a todo el programa de pensamiento crítico de la liberación de Dussel, de lo contrario nos perderemos en el bosque. En la primera parte hay una diferencia entre lo moral y lo crítico, no está de más recordar que apostamos por lo segundo. Lo primero se convierte en funcional al sistema vigente, diríamos que al capitalismo. Lo segundo, entonces, es la proximidad del caracara, es "recuperar al Otro/a como distinto del sistema que lo ha cosificado" (Dussel, 2016: 13). La segunda parte es la propositiva. Como ya hemos dicho, y Dussel siempre lo recuerda, la Ética es una teoría general de los campos prácticos. Esta versión de la Ética quedaría entre los dos tomos de la Política y el tercero que cerraría esa trilogía. Podríamos leer esta segunda parte de las 14 tesis de ética como apertura de un pensamiento crítico de la liberación propositivo. Que va de la deconstrucción del orden vigente hasta la creatividad de la formación de un orden alternativo, mediante una praxis de la liberación.

\section{Bibliografía}

APEL, K.-O., DUSSEL, E. y BETANCOURT. (1992). Fundamentación de la ética y filosofía de la liberación. México: Siglo XXI / UAM-Itzapala.

ARROYO, F. (23-4-2016). Giorgio Agamben: "El ciudadano es para el Estado un terrorista virtual". El País.

BAUTISTA, J. J. (2014). ¿Qué significa pensar desde América Latina? Madrid: Akal.

BEORLEGUI, C. (2010). Historia del pensamiento filosófico latinoamericano. Una búsqueda incesante de la identidad. Bilbao: Universidad de Deusto.

CALVINO, I. (2009). Por qué leer los clásicos. Madrid: Siruela.

CALVO, J. L. (1994). (Introducción, traducción y notas). Aristóteles. Ética a Nicómaco. Madrid: Alianza.

CARBONARI, P. C., DA COSTA, J. A. y MACHADO, L., 2015. Filosofia e Libertação Homenagem aos 80 anos de Enrique Dussel. Passo Fundo: Instituto Superior de Filosofia Berthier.

CERUTTI, H. (2006). Filosofía de la liberación latinoamericana. México: Fondo de Cultura Económica.

DE SOUSA SANTOS, B. (2009). Una epistemología del sur. La reinvención del conocimiento y la emancipación social. México: CLACSO / Siglo XXI. 
DUSSEL, E. (1966). Hipótesis para el estudio de Latinoamérica en la historia universal: (Investigación del mundo donde se constituyen y evolucionan las weltanschauungen). Resistencia.

DUSSEL, E. (1969). El humanismo semita. Estructuras radicales del pueblo de Israel y otros semitas. Buenos Aires: EUDEBA.

DUSSEL, E. (1970). Para una de-strucción de la historia de la ética. Mendoza: Ser y Tiempo.

DUSSEL, E. (1974a). Historia de la iglesia en América Latina. Coloniaje y liberación 1492/1973. Barcelona: Nova Terra.

DUSSEL, E. (1974b). Método para una filosofía de la liberación. Superación analéctica de la dialéctica hegeliana. Salamanca: Ediciones Sígueme.

DUSSEL, E. (1984). Filosofía de la producción. Bogotá: Nueva América.

DUSSEL, E. (1988). Hacia un Marx desconocido. Un comentario de los Manucritos del 61-63. México: Siglo XXI.

DUSSEL, E. (1990). El último Marx (1863-1882) y la liberación latinoamericana. Un comentario a la tercera y a la cuarta redacción de "El capital". México: Siglo XXI.

DUSSEL, E. (1991²). La producción teórica de Marx. Un comentario a los Grundrisse. México: Siglo XXI.

DUSSEL, E. (1992). Historia de la iglesia en América Latina. Medio milenio de coloniaje y liberación (1492-1992). Madrid: Mundo Negro - Esquila Misional.

DUSSEL, E. (1993a). Apel, Ricoeur, Rorty y la filosofía de la liberación con respuestas de Karl-Otto Apel y Paul Ricoeur. Guadalajara: Universidad de Guadalajara.

DUSSEL, E. (1993b). Las metáforas teológicas de Marx. Navarra: Verbo Divino.

DUSSEL, E. (1998a). Ética de la liberación ante el desafio de Apel, Taylor y Vattimo con respuesta inédita de K.-O. Apel. México: UAEM.

DUSSEL, E. (1998b). Ética de la Liberación en la edad de la globalización y de la exclusión. Madrid: Trotta.

DUSSEL, E. (2001). Hacia una filosofía política crítica. Bilbao: Desclée.

DUSSEL, E. (2006). 20 tesis de política. México: CREFAL / Siglo XXI.

DUSSEL, E. (2007a). Materiales para una política de la liberación. Madrid: Plaza y Valdés.

DUSSEL, E. (2007b). Política de la liberación. Historia mundial y crítica. Madrid: Trotta.

DUSSEL, E. (2009). Política de la liberación. Volumen II arquitectónica. Madrid: Trotta.

DUSSEL, E. (2011). Filosofía de la Liberación. México: Fondo de Cultura Económica.

DUSSEL, E. (2014a). 16 tesis de economía política. Interpretación filosófica. México: Siglo XXI.

DUSSEL, E. (2014b). Para una Ética de la Liberación Latinoamericana. México: Siglo XXI. 
DUSSEL, E. (2015). Filosofías del Sur. Descolonización y Transmodernidad. México: Akal.

DUSSEL, E. (2016). 14 tesis de ética. Hacia la esencia del pensamiento crítico. Madrid: Trotta.

DUSSEL, E., MENDIETA, E. y BOHÓRQUEZ, C. (2011). El pensamiento filosófico latinoamericano, del Caribe y "latino" 1300-2000. México: Siglo XXI.

FORNET-BETANCOURT, R. (2004). Crítica intercultural de la filosofía latinoamericana actual. Madrid: Trotta.

GARCÍA, M. (1988). (Introducción, traducción y notas). Aristóteles. Política. Madrid: Gredos.

HALL, S. (2008). ¿Cuándo fue lo postcolonial? Pensar al límite. En S. Mezzadra (Ed.), Estudios postcoloniales Ensayos fundamentales (págs. 121-144). Madrid: Traficantes de Sueño.

HARDT, M. (2010). Política y multidud. En I Ciclo de Conferencias Internacionales. Pensando el Mundo desde Bolivia (págs. 65-74). La Paz: Vicepresidencia Del Estado Plurinacional de Bolivia.

HILL, P. (2000). Black Feminist Thought. New York and London: Routledge.

MALDONADO-TORRES, N. (2006). Césaire's Gift and the Decolonial Turn. Radical Philosophy Review, 9 (2), pp. 111-137.

MALDONADO-TORRES, N. (2008). Against War: Views from the Underside of Modernity. Durham: Duke University Press.

MARTÍN ALCOFF, L. y MENDIETA, E. (2000). Thinking from the Underside of History. Enrique Dussel's Philosophy of Liberation. Unite State of America: Bowman \& Littefield Publishers.

MARX, K. (1971). Diferencia de la filosofía de la naturaleza en Demócrito y en Epicuro. Madrid: Editorial Ayuso.

MENESES, M. P. y DE SOUSA SANTOS, B. (2014). Espistemologías del sur (Perspectivas). Madrid: Akal.

MIGNOLO, W. (2003). The Darker Side of the Renaissance. Literacy, Territoriality \& Colonization. Michigan: University of Michigan Press.

MORENO, M. (1998). Cronología de Enrique Dussel. Anthropos. Huellas del conocimiento (180), 37-46.

MYERS, J. (2008). Introducción al volumen 1. Los intelectuales latinoamericanos desde la colonia hasta el inicio del siglo XX. En C. Altamirano (Ed.), Historia de los intelectuales en América Latina. 1. La ciudad letrada, de la conquista al modernismo (págs. 29-50). Buenos Aires: Katz.

RICHTER, D. (2011). El Sur. Historia de un punto cardinal. Madrid: Siruela.

ROBINDSON, C. (2000). Black Marxism. The United State of America: The University of North Carolina Press.

SASTRE, A. (2005). La batalla de los intelectuales: o nuevo discurso de las armas y las letras. Buenos Aires: CLACSO.

WALLERSTEIN, I. (2000). El eurocentrismo y sus avatares: los dilemas de las ciencias sociales. New Left Review (0), 97-113.

ZIZEK, S. y SRECKO, H. (2014). El sur pide la palabra. Barcelona: Libros del Lince. 